\title{
Performance of breast magnetic resonance imaging in axillary nodal staging in newly diagnosed breast cancer patients
}

\author{
Jatuporn Chayakulkheeree ${ }^{A, C, D, E}$, Dirapit Pungrassami ${ }^{B}$, Jenjeera Prueksadee ${ }^{F}$ \\ Division of Diagnostic Radiology, Department of Radiology, Faculty of Medicine Chulalongkorn University, Thailand
}

\section{Abstract}

\begin{abstract}
Purpose: To determine the diagnostic value of breast magnetic resonance imaging (MRI) in detecting axillary metastatic node in newly diagnosed breast cancer, we assessed the sensitivity, specificity, positive predictive value (PPV), and negative predictive value (NPV) of breast MRI.
\end{abstract}

Material and methods: Data were collected retrospectively from January 2011 to December 2018. Preoperative breast MRI of women with newly diagnosed breast cancer were reviewed to evaluate the axillary nodal staging by using the morphological characteristic of metastatic axillary lymph node. Statistical analysis was performed to assess the performance of breast MRI in diagnosing metastatic axillary lymph nodes based on the pathological result.

Results: A total of 131 women with breast cancer were analysed. Two hundred and twenty-seven axillary lymph nodes from preoperative breast MRIs were considered to be metastasis. $65.65 \%$ (86 patients) of the breast MRI results matched with the pathological results: 37 patients with N0 stage, 39 patients with N1 stage, eight patients with N2 stage, and two patients with N3 stage. Sensitivity of breast MRI for axillary nodal staging was 98.5\% (95\% CI: 92-100\%), and the negative predictive value was $96.4 \%$ (86.2-99.9\%). Specificity of breast MRI for axillary nodal staging was $57.8 \%(44.8-70.1 \%)$ and the positive predictive value was $71 \%(60.6-79.9 \%)$.

Conclusions: Our study showed that the breast MRI had a high sensitivity (98.5\%) and high NPV (96.4\%) in detecting metastatic axillary lymph nodes, but its specificity was only fair (57.8\%). Overestimation and underestimation of the MRI in N staging were also found in $20.61 \%$ and $12.98 \%$ of cases, respectively.

Key words: breast cancer, axilla, lymph nodes, metastasis, magnetic resonance imaging, breast MRI.

\section{Introduction}

The axillary nodal status is one of the most important prognostic factors in patients with invasive breast cancer because it can help doctors choose the most appropriate treatment (i.e. systemic chemotherapy and/or radiation therapy) for their patients. Sentinel lymph node biopsy (SLNB) is the primary method used to exclude axillary metastases in most cases with early stage breast cancer. A positive SLNB result indicates that the patient will usually undergo axillary lymph node dissection (ALND) with removal of level I and II axillary nodes [1]. The National Comprehensive Cancer Network (NCCN) guideline recommends radiation therapy to the whole breast or chest wall and regional lymph nodes in woman with positive lymph nodes [2]. Thus, prediction of nodal metastasis in preoperative breast cancer is influential in the treatment plan.

Breast magnetic resonance imaging (MRI) can be utilised to evaluate the extension of the breast cancer and affected area such as the skin, pectoral muscle, or axillary lymph nodes, which are important for prognosis [3]. Morphological features with metastatic node, for example cortical thickening, loss of fatty hilum, heterogeneous enhancement, and round shape or a long to short axis ratio of less than 2, are considered suspicious. In prior studies, the presence of any axillary node with no fatty hilum seen

\section{Correspondence address:}

Dr. Jatuporn Chayakulkheeree, Department of Radiology, Faculty of Medicine, Chulalongkorn University, Bangkok, Thailand, e-mail: Jatuporn.@@chula.ac.th

Authors' contribution:

A Study design · B Data collection · C Statistical analysis · D Data interpretation · E Manuscript preparation · F Literature search · G Funds collection 


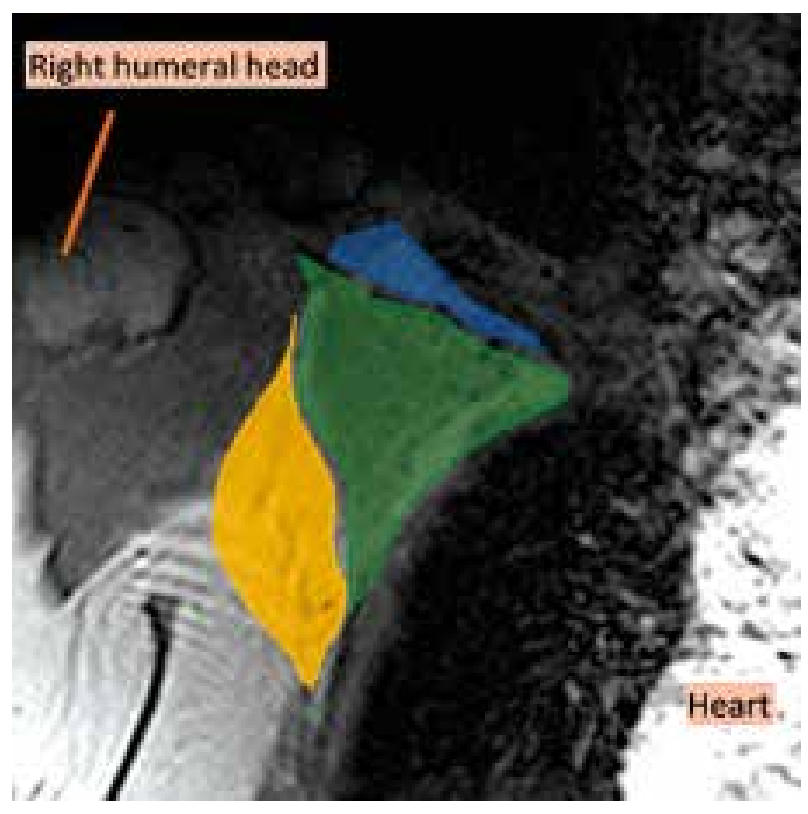

Figure 1. Coronal magnetic resonance imaging (MRI) of the right axillary region, T2WI. The axilla is divided into three levels on the MRI in the coronal plane. Level I was inferolateral to the pectoralis minor muscle (yellow), level II was behind the pectoralis minor muscle (green), and level III was superomedial to the pectoralis minor muscle (blue)

on MRI and with a high signal intensity on T2 sequences and marked gadolinium enhancement were found to be significantly correlated with metastatic lymph nodes $[4,5]$.

As a result of this, we assessed the performance of breast MRI in evaluating axillary nodal staging in newly diagnosed breast cancer patients by using morphological characteristics of metastatic axillary lymph node.

\section{Material and methods}

\section{Study population}

We retrospectively reviewed preoperative breast MRIs of newly diagnosed breast cancer patients who underwent

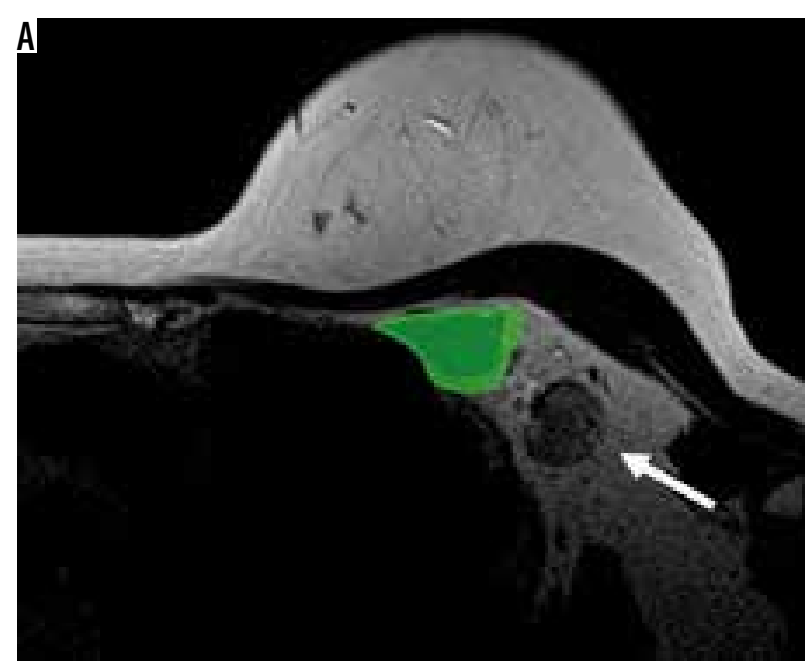

breast MRI at our academic institution from January 2011 to December 2018. All patients underwent SLNB or ALND, and the pathological nodal stage was obtained. Timing from performing MRI to surgery was within two months.

Patients with carcinoma in situ (ductal or lobular; DCIS or LCIS), who previously had adjuvant chemotherapy or whole breast radiation, and who had known breast cancer or prior axillary surgery were excluded from the study. This left us with a total of 131 patients. This study was approved by the Ethics Committee of the Faculty of Medicine, Chulalongkorn University.

\section{Magnetic resonance imaging protocols}

MRI was performed using a 1.5-Tesla system (Siemen Magnetom Espree, Siemens Medical Solutions, Erlangen, Germany) with an eight-channel breast coil.

The pre-contrast sequences include axial three-dimensional high-resolution, fat-suppressed, T1-weighted, axial, short-T1 inversion recovery (STIR), axial T2-weighted images turbo-spin echo (TSE), and coronal T1-weighted sequences.

After the contrast injection, dynamic contrast-enhanced MRI (DCE-MR imaging) was performed with axial three-dimensional high-resolution T1-weighted, axial three-dimensional fat-suppressed T1-weighted with and without subtraction, DWI, ADC, sagittal T2-weighted turbo spin echo, and coronal $\mathrm{T} 2$-weighted sequences.

\section{Magnetic resonance imaging evaluation}

The axillary lymph nodes were divided into three levels by the pectoralis minor muscle as follows: level I was inferolateral to the pectoralis minor muscle, level II was behind the pectoralis minor muscles, and level III was superomedial to the pectoralis minor muscle [3] (Figures 1 and 2). All nodes were assessed by using axial T2-weight without

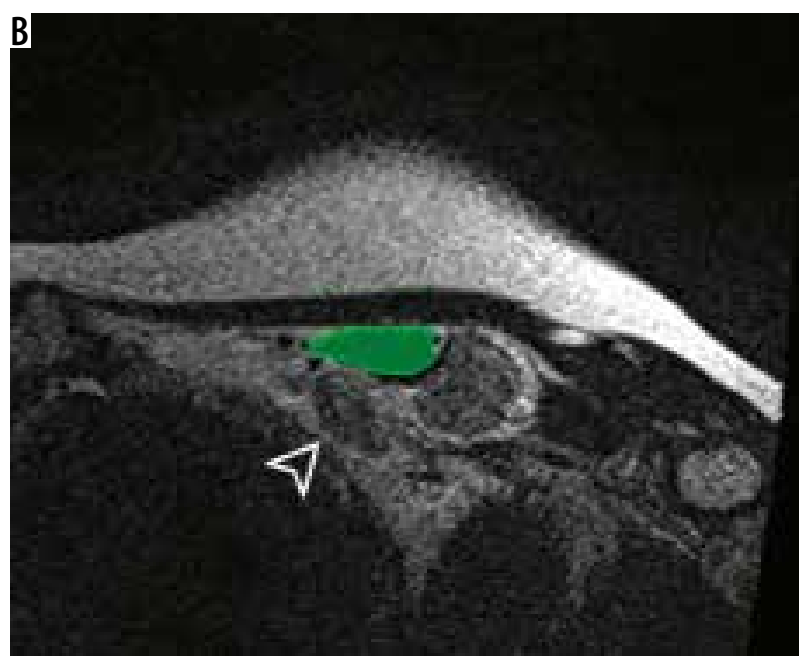

Figure 2. Axillary level I (A) and II (B) on magnetic resonance imaging axial T2WI. The axillary lymph node level I (arrow) is located lateral to the pectoralis minor muscle (green) while the axillary level II lymph node (arrowhead) is located behind the pectoralis minor muscle (green) 

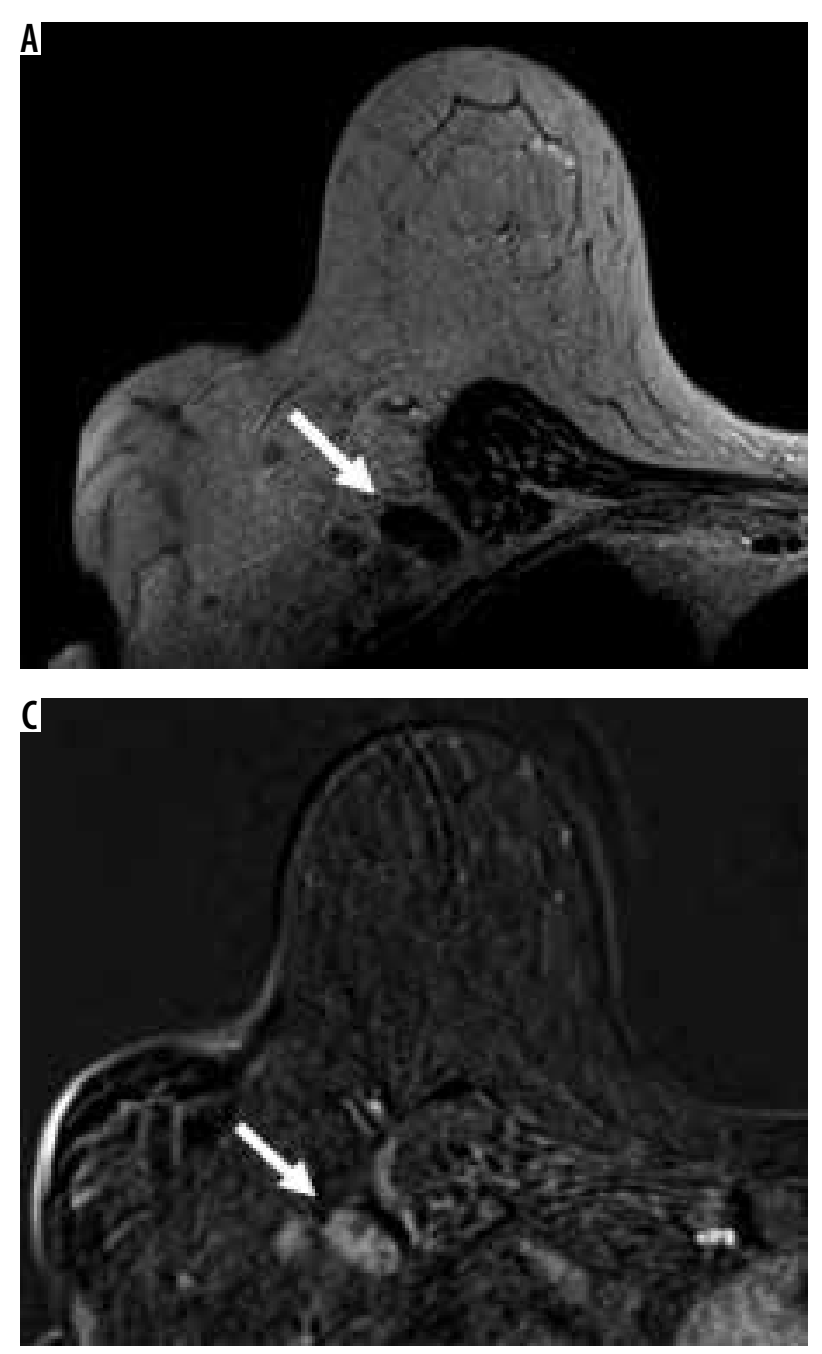

fat suppression, post contrast T1-weight sequence, and coronal T2-weighted sequences.

All MRI data were separately evaluated by two radiologists who had more than 11 years' experience in reading breast images. The observers were blinded to the patients' information, radiological reports, and histopathological information to avoid bias. Consensus was made in cases of disagreement.

Morphology criteria of pathologic nodes were considered when there were two of three features of cortical thickening greater than $2 \mathrm{~mm}$, short axis size greater than $5 \mathrm{~mm}$, and long to short axis ratio less than two. Signal intensity on T2-weighted image and pattern of enhancement were also collected. $\mathrm{N}$ staging was determined based on the number of metastatic axillary lymph nodes as follows: N0 - no regional lymph node metastasis, N1 - metastases in 1-3 axillary lymph nodes, N2 - metastases in 4-9 axillary lymph nodes, and N3 - metastases in 10 or more axillary lymph nodes.

\section{Pathologic nodal staging}

According to the American Joint Committee on Cancer (AJCC), pathological results were determined by

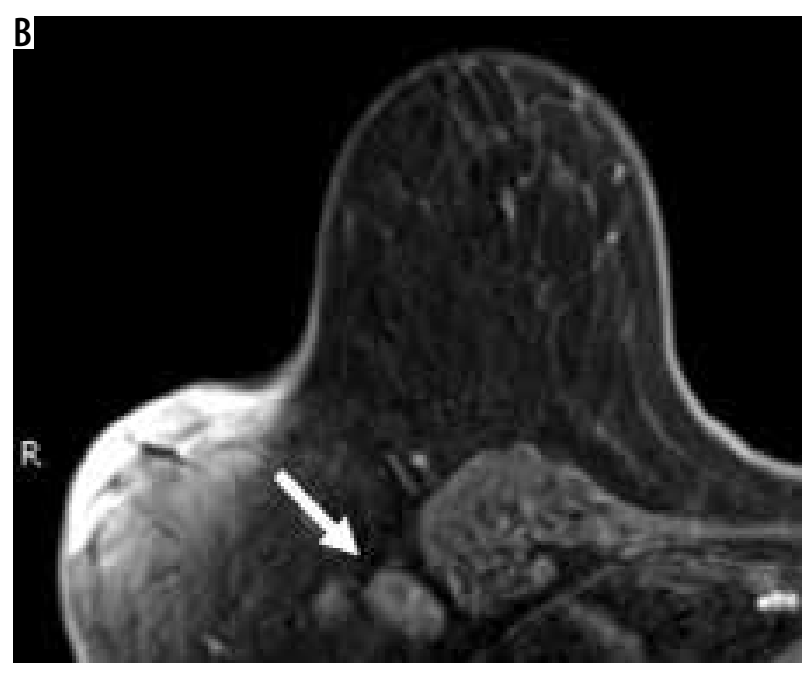

Figure 3. A 70-year-old female with right breast cancer. Axial magnetic resonance imaging (MRI) of the right breast showed an enlarged level I axillary lymph node without fatty hilum and thickened cortex (arrow). From the breast MRI, there was a hyper signal intensity on T2-weighted image (A) with heterogeneous enhancement on contrast-enhanced T1-weighted image with fat suppression (B) and with subtraction (C), which looked suspiciously like a metastatic lymph node. This patient has pN3 stage from pathology

the number of proven metastatic axillary lymph nodes based on the final pathologic results of the surgical specimens from SLNB or ALND, as follows: pN0 - histologically no regional lymph node metastasis, $\mathrm{pN} 1$ micrometastases or metastases in 1-3 axillary lymph nodes, pN2 - metastases in 4-9 axillary lymph nodes, and $\mathrm{pN} 3$ - metastases in 10 or more axillary lymph nodes. However, the axillary lymph node dissection was performed on level I and II axillary lymph nodes; therefore, our pathological surgical specimens were limited to just these levels (Figure 3).

\section{Statistical analysis}

The mean \pm SD was used for continuous data such as age, interval between preoperative breast MRI and surgical treatment, short axis, long axis, and long to short axis ratio. Other demographic data and MRI characteristic were evaluated using percentages.

Sensitivity, specificity, positive predictive value, and negative predictive value were computed on $2 \times 2$ tables of true-positive, true-negative, false-positive, and false-negative values. Subgroup analyses of the different nodal staging were performed. 
The $\kappa$ coefficient was analysed to assess how well the diagnoses of metastatic axillary lymph nodes based on MRI interpretation matched those based on pathologic evaluation at the pathological $\mathrm{N}$ staging. The strength of agreement beyond chance for various ranges of $\kappa$ that were suggested by Landis and Koch [6] were used: $\kappa<0-$ poor, $\kappa=0.00-0.20-$ slight, $\kappa=0.21-0.40-$ fair, $\kappa=0.41-0.60-$ moderate, $\kappa=0.61-0.80-$ substantial, and $\kappa=0.81-1.00$ - almost perfect.

\section{Results}

During the period from January 2011 to December 2018, 131 women with breast cancer had preoperative breast MRI. The mean age of the patients was 51.33 years (range:

Table 1. Demographics of the study patients $(n=131)$

\begin{tabular}{|l|c|c|}
$\begin{array}{l}\text { Characteristic } \\
(n=131)\end{array}$ & $\begin{array}{c}\text { Mean } \pm \text { SD } \\
\text { or } n(\%)\end{array}$ & $\begin{array}{c}\text { Median } \\
\text { [min, max] }\end{array}$ \\
\hline Age & $51.33 \pm 10.17$ & $50[28,84]$ \\
\hline \begin{tabular}{l} 
Site \\
\hline Right
\end{tabular} & $72(55 \%)$ & \\
\hline Left & $59(45 \%)$ & \\
\hline Surgery & & \\
\hline $\begin{array}{l}\text { Wide excision, axillary node } \\
\text { dissection }\end{array}$ & $52(39.7 \%)$ & \\
\hline Modified radical mastectomy & $73(55.7 \%)$ & \\
\hline Sentinel node biopsy & $6(4.6 \%)$ & \\
\hline Histopathologic type & $118(90.1 \%)$ & \\
\hline IDC & $5(3.8 \%)$ & \\
\hline ILC & $8(6.1 \%)$ & \\
\hline Other* & $36.13 \pm 36.18$ & $25[1,203]$ \\
\hline Interval (day) & & \\
\hline Pathological N staging & $64(48.9 \%)$ & \\
\hline pN0 & $39(29.8 \%)$ & \\
\hline pN1 & $18(13.7 \%)$ & \\
\hline pN2 & $10(7.6 \%)$ & \\
\hline pN3 & $38(29.0 \%)$ & \\
\hline MRI results & $80(61.1 \%)$ & \\
\hline N0 & $11(8.4 \%)$ & \\
\hline N1 & $2(1.5 \%)$ & \\
\hline N2 & & \\
\hline N3 & & \\
\hline
\end{tabular}

IDC - invasive ducal carcinoma, ILC - invasive lobular carcinoma

*0ther subtypes of breast cancer

Pathological N staging: pNO - no regional lymph node metastasis histologically, pN1 - micrometastases; or metastases in 1-3 axillary lymph nodes, pN2 - metastases in 4-9 axillary lymph nodes, pN3 - metastases in 10 or more axillary lymph nodes

$\mathrm{MRI}$ results ( $\mathrm{N}$ staging): $\mathrm{N} 0$ - no regional lymph node metastasis, $\mathrm{N} 1$ - metastases in 1-3 axillary lymph nodes, N2 - metastases in 4-9 axillary lymph nodes, N3 - metastases in 10 or more axillary lymph nodes
28 to 84 years). All women underwent surgery as follows: $52(39.7 \%)$ patients had wide excision, axillary lymph node dissection; 73 (55.7\%) patients had modified radical mastectomy; and six (4.6\%) patients had sentinel node dissection. The mean interval between preoperative breast MRI and surgery was 36.13 days. The histopathological type of primary tumour was invasive ductal carcinoma metastasis ( $n=118 ; 90.1 \%)$, invasive lobular carcinoma metastasis $(n=5 ; 3.8 \%)$, and other metastasis $(n=8$; $6.1 \%$ ) (Table 1).

According to the pathologic results, 64 women (48.9\%) had negative metastatic axillary lymph nodes (pN0), 39 women (29.8\%) had metastases in 1-3 axillary lymph nodes (pN1), 18 women (13.7\%) had metastases in 4-9 axillary lymph nodes ( $\mathrm{pN} 2$ ), and 10 women (7.6\%) had metastases in 10 or more axillary lymph nodes ( $\mathrm{pN} 3$ ) (Table 1).

A total of 227 axillary lymph nodes were analysed from preoperative breast MRI, which were considered to be metastatic nodes. 193 (85\%) nodes were level I (inferolateral to the pectoralis minor muscle), 34 (15\%) nodes were level II (behind the pectoralis minor muscles), and $0(0 \%)$ nodes were level III (superomedial to the pectoralis minor muscle).

The long axis ranged from 0.5 to $3.3 \mathrm{~cm}$ (average 1.22 $\pm 0.50 \mathrm{~cm}$ ), and the short axis ranged from 0.4 to $2.4 \mathrm{~cm}$ (average $0.79 \pm 0.36 \mathrm{~cm}$ ). The long to short axis ratio measurements of the lymph nodes ranged from 1.05 to 3.75 (average $1.59 \pm 0.35$ ) (Table 2).

Table 2. MRI characteristics of the axillary lymph nodes $(n=227)$

\begin{tabular}{|l|c|c|}
\hline $\begin{array}{l}\text { Site } \\
\text { Right }\end{array}$ & $\begin{array}{c}\text { Median } \\
\text { Mean } \pm \text { SD }\end{array}$ & \\
\hline Left & $109(48 \%)$ & \\
\hline \begin{tabular}{l} 
Level \\
\hline Level I
\end{tabular} & $118(52 \%)$ & \\
\hline Level II & $193(85 \%)$ & \\
\hline Level III & $34(15 \%)$ & \\
\hline Fatty hilum & $0(0 \%)$ & \\
\hline Absence & $179(78.9 \%)$ & \\
\hline Presence & $48(21.1 \%)$ & \\
\hline Long axis & $1.22 \pm 0.50$ & $1.1[0.5,3.3]$ \\
\hline Short axis & $0.79 \pm 0.36$ & $0.7[0.4,2.4]$ \\
\hline L/S ratio & $1.59 \pm 0.35$ & $1.55[1.05,3.75]$ \\
\hline Hyper intense T2 & & \\
\hline Presence & $227(100 \%)$ & \\
\hline Enhancement & & \\
\hline Homogeneous & $92(40.5 \%)$ & \\
\hline Heterogeneous & $135(59.5 \%)$ & \\
\hline
\end{tabular}


Table 3. Correlation of breast MRI with pathological axillary nodal staging

\begin{tabular}{|l|c|c|c|c|c|}
\hline \multirow{2}{*}{ MRI N staging } & \multicolumn{5}{|c|}{ Pathological N staging } \\
\cline { 2 - 6 } & $\mathrm{pN0}$ & $\mathrm{pN1}$ & $\mathrm{pN2}$ & $\mathrm{pN3}$ & Total \\
\hline N0 & 37 & 0 & 1 & 0 & 38 \\
\hline N1 & 27 & 39 & 9 & 5 & 80 \\
\hline N2 & 0 & 0 & 8 & 3 & 11 \\
\hline N3 & 0 & 0 & 0 & 2 & 2 \\
\hline Total & 64 & 39 & 18 & 10 & 131 \\
\hline
\end{tabular}

Pathological N staging: pN0 - no regional lymph node metastasis histologically, pN1 - micrometastases; or metastases in 1-3 axillary lymph nodes, pN2 - metastases in 4-9 axillary lymph nodes, pN3 - metastases in 10 or more axillary lymph nodes

MRI results (N staging): N0 - no regional lymph node metastasis, N1 - metastases in 1-3 axillary lymph nodes, N2 - metastases in 4-9 axillary lymph nodes, N3 - metastases in 10 or more axillary lymph nodes

Absent fatty hilum was observed in 179 metastatic axillary lymph nodes $(78.9 \%)$, and fatty hilum was seen in 34 metastatic axillary lymph nodes $(21.1 \%)$. All metastatic nodes showed hyper signal intensity on T2-weighted image. $40.5 \%(n=92)$ were homogeneous and $59.59 \%$ $(n=135)$ were heterogenous (Table 2$)$. On the MRI, our true positive case that was considered to have metastatic axillary node did have a positive lymph node in the final pathology.

In the subgroup analysis of $\mathrm{N}$ staging, our study also demonstrated that $65.65 \%$ ( 86 out of 131 patients) of the MRI results matched with the pathological results. Thirtyseven of 64 patients (57.81\%) had N0 stage, 39 of 39 patients (100\%) had N1 stage, eight of 18 patients $(44.44 \%)$ had N2 stage, and two of 10 patients (2\%) had N3 stage (Table 3).

The sensitivity of breast MRI for detecting metastatic axillary lymph node was $98.5 \%$ (95\% CI: 92-100\%), and the negative predictive value was $96.4 \%$ (86.2-99.9\%). The specificity of breast MRI for detecting metastatic axillary lymph node was $57.8 \%(44.8-70.1 \%)$, and the positive predictive value was $71 \%$ (60.6-79.9\%).

The $\kappa$ coefficient was analysed to assess the correlation of breast MRI with pathological axillary nodal staging. It was shown that there was a moderate agreement beyond chance $(\kappa=0.48,95 \% \mathrm{CI}: 0.386-0.539)$.

\section{Discussion}

Breast MRI is widely used for preoperative assessment for extension of breast cancer, axillary lymph node status, and screening of the contralateral breast in breast cancer patients [7]. The advantages of breast MRI over other imaging modalities are its accuracy for providing anatomical information of the breast and axilla.

In our study, 227 MRIs that were considered as metastasis axillary lymph nodes were collected from 131 women with breast cancer. The sensitivity, specificity, negative predictive value, and positive predictive value for detect- ing axillary nodal metastasis in breast cancer were $98.5 \%$, $57.8 \%, 96.4 \%$, and $71 \%$, respectively. Our results support the findings from previous studies that assessed the diagnostic accuracy of breast MRI for detecting axillary nodal metastasis in breast cancer patients $[8,9]$. He et al. and Li et al. demonstrated high sensitivity (97\% and $94.6 \%$, respectively), and high negative predictive value (99.4\% and $99.5 \%$, respectively) of MRI to detect axillary lymph node metastasis in patients with breast cancer $[8,9]$.

Despite the high sensitivity of breast MRI for determining metastatic nodes, overestimated or underestimated $\mathrm{N}$ staging can be found. In our data, there were overestimated and underestimated MRIs of $\mathrm{N}$ staging in 27 out of 131 patients $(20.61 \%)$ and in 17 out of 131 patients (12.98\%), respectively.

However, breast MRI could detect early nodal metastasis in breast cancer patients, especially N1 stage. Early detection of nodal metastasis is very helpful in designing the treatment plan for the patient.

A limitation of our study is that it was a retrospective study. In our study, we could not compare level by level or node by node of the MRI positive nodes matched to the pathologic positive nodes.

\section{Conclusions}

In conclusion, our study showed high sensitivity (98.5\%) and high negative predictive value (96.4\%) of breast MRI in detecting metastatic axillary lymph nodes, but its specificity was only fair (57.8\%). $20.61 \%$ of the MRIs overestimated the $\mathrm{N}$ staging, and $12.98 \%$ of the MRIs underestimated the $\mathrm{N}$ staging. Nevertheless, we found high performance of MRI in detecting the N1 stage, which is very helpful in designing the treatment plan for patients with early nodal metastases ( $\mathrm{pN} 1)$.

\section{Conflict of interest}

The authors report no conflict of interest. 


\section{References}

1. Ecanow JS, Abe H, Newstead GM, Ecanow DB, Jeske JM. Axillary staging of breast cancer: what the radiologist should know. Radiographics 2013; 33: 1589-1612.

2. Gradishar WJ, Anderson BO, Balassanian R, et al. Breast cancer. Version 3.2018, NCCN Clinical Practice Guidelines in Oncology 2018.

3. Arslan G, Altintoprak KM, Yirgin IK, et al. Diagnostic accuracy of metastatic axillary lymph nodes in breast MRI. Springerplus 2016; 5: 735.

4. Mortellaro VE, Marshall J, Singer L, et al. Magnetic resonance imaging for axillary staging in patients with breast cancer. J Magn Reson Imaging 2009; 30: 309-312.

5. Luciani A, Dao TH, Lapeyre M, et al. Simultaneous bilateral breast and high-resolution axillary MRI of patients with breast cancer: preliminary results. AJR Am J Roentgenol 2004; 182: 1059-1067.
6. Landis JR, Koch GG. The measurement of observer agreement for categorical data. Biometric 1997; 33: 159-174.

7. Lehman CD, DeMartini W, Anderson BO, Edge SB. Indications for breast MRI in the patient with newly diagnosed breast cancer. J Natl Compr Cancer Netw 2009; 7: 193-201.

8. He N, Xie C, Wei W, et al. A new, preoperative, MRI-based scoring system for diagnosing malignant axillary lymph nodes in women evaluated for breast cancer. Eur J Radiol 2012; 81: 2602-2612.

9. Li C, Meng S, Yang X, et al. The value of T2* in differentiating metastatic from benign axillary lymph nodes in patients with breast cancer - a preliminary in vivo study. PLoS One 2014; 9: e84038. 